\title{
TREES AND DISCRETE SUBGROUPS OF LIE GROUPS OVER LOCAL FIELDS
}

\author{
ALEXANDER LUBOTZKY
}

Let $K$ be a locally compact field and $\mathbf{G}$ a simple $K$-group, $G=\mathbf{G}(K)$. A discrete subgroup $\Gamma$ of $G$ is called a lattice if $G / \Gamma$ carries a finite $G$-invariant measure. It is a uniform (or cocompact) lattice if $G / \Gamma$ is compact and nonuniform otherwise.

When the $K$-rank of $G$ is greater than one, Margulis [Ma, Z] proved that $\Gamma$ is arithmetic, establishing the conjecture of Selberg and PiatetskiShapiro. This remarkable work left open the case of rank one groups. $S L_{2}(\mathbb{R})$ contains continuous families of lattices (the Teichmüller spaces) and in particular it contains nonarithmetic lattices. By the Mostow rigidity theorem (cf. [M1]), $S L_{2}(\mathbb{R})$ is essentially the only real simple Lie group which allows this phenomenon. Some other real rank one groups are known to have nonarithmetic lattices: Gromov and Piatetski-Shapiro [GPS] showed that $S O(n, 1)$ have such lattices (earlier it was shown by Makarov and Vinberg for small $n$ ), Mostow [M2] constructed nonarithmetic lattices in $S U(2,1)$, and together with Deligne [DM] also in $S U(3,1)$. For the other rank one real groups: $S U(n, 1)(n \geq 4), S p(n, 1)$ and $F_{4}$ the problem is still open.

A related problem is the congruence subgroup problem, which asked: Given $\Gamma$ arithmetic, are all its finite index subgroups congruence subgroups? Serre [S1] conjectured that $\Gamma$ has the congruence subgroup property (CSP) if and only if $\operatorname{rank}_{K}(G) \geq 2$. The affirmative part of this conjecture was proved to a large extent (but mainly for nonuniform lattices; see [R1, R2] for precise results and history). Less is known for arithmetic lattices in real rank one groups: It is easy to prove that none of the arithmetic lattices in $P_{2} L_{2}(\mathbb{R})=S O(2,1)^{0}$ has CSP. The same holds for $P S L_{2}(\mathbb{C})=S O(3,1)$ (see [S1] for the nonuniform case and [L1] for the uniform case). For $S O(n, 1)$, general $n$, it is known only for some of the lattices (Millson [Mi]). Similarly, Kazdhan [Ka] showed that some lattices in $S U(n, 1)$ do not have CSP. Again, nothing is known for $S p(n, 1)$ and $F_{4}$, which are of rank one but have Kazdhan property (T) like groups of higher rank.

In this note we will describe results on the structure of lattices in rank one groups over locally compact nonarchimedean fields, as well as some

Received by the editors May 24, 1988. $20 \mathrm{G} 25$.

1980 Mathematics Subject Classification (1985 Revision). Primary 22E40; Secondary

Key words and phrases. Lie groups over local fields, discrete subgroups, trees. 
constructions of such lattices. The bottom line of our work is that regarding discrete subgroups all these groups are similar to $S L_{2}(\mathbb{R})$ : They allow deformation spaces of lattices and in particular have nonarithmetic lattices. We also prove Serre's conjecture that all the arithmetic lattices in such groups fail to satisfy the congruence subgroup property.

From now on, let $\mathbf{G}$ be a rank one almost simple algebraic group defined over a locally compact nonarchimedean field $K$, and $G=\mathbf{G}(K)$. Associated with $G$ there is a tree $X$ (the Bruhat-Tits building) on which $G$ acts with two orbits of vertices and transitively on edges [BT].

THeOREM 1. G has an uncountable number of conjugacy classes of cocompact lattices and in particular nonarithmetic lattices. If char $K>0$ then the same holds with nonuniform lattices.

Recall that when char $K=0$ there are no nonuniform lattices (cf. [S2, p. 84]).

Theorem 1 is proved by making two constructions, which use heavily the action of $G$ on the tree and its boundary.

1st construction: Schottky groups. Here one imitates the classical construction of Schottky groups acting on the unit disc model of the hyperbolic plane. A similar construction using properties of $S L_{2}$ is presented in [GP]. But we rather present the construction in a purely combinatorial way which enables us to do it in any group of automorphisms of a tree, in particular in rank one groups over $K$.

Schottky groups may have infinite or finite fundamental domain, but if they are lattices, they are necessarily cocompact. To get nonuniform lattices in case char $K=p>0$ we have

2nd construction: Cusp subgroups. Here we want to construct groups with infinite fundamental domain on $X$ but with finite volume. See the group $\Gamma=S L_{2}(k[t]) \subseteq G=S L_{2}(k((1 / t)))$ discussed in [S2, p. 87]. For simplicity we present the construction here only for this $G$. The case of rank one groups in which the unipotent radical $U$ of a minimal parabolic subgroup $B$ is abelian can be handled in exactly the same way, but the general case (i.e., $U$ is two step nilpotent) needs more arguments for which the reader is referred to [L2].

Let

$$
U=\left(\begin{array}{cc}
1 & K \\
0 & 1
\end{array}\right) \subset G \text { and } \Delta=\left(\begin{array}{cc}
1 & t k[t] \\
0 & 1
\end{array}\right)
$$

Then $\Delta$ is a lattice in $U$. The boundary of $X$ can be identified with the spherical building associated to $G$, i.e., $G / B \simeq U \cup\{\infty\}$. In our case this is just $P^{1}(K)$. One can easily compute that a fundamental domain for $\Delta$ on $X$ is of the following type: Let $X_{0}$ be a fixed point (say the standard $k[[1 / t]]-$ module), $L=\left\{X_{0}, X_{1}, X_{2}, \ldots\right\}$ the sequence of points going from $X_{0}$ to the point $\infty$ on the boundary. Let $X_{1}, Y_{1}, \ldots, Y_{p}$ be the neighbors of $X_{0}$ and $E_{i}=\left\{x \in X \mid d\left(x, Y_{i}\right)<d\left(x, X_{0}\right)\right\}$ for $i=1, \ldots, p$. Then $F=\left(\bigcup_{i=1}^{p} E_{i}\right) \cup L$ is a fundamental domain for $\Delta$ on $X$.

Now, let $g_{0}=\mathrm{id}, g_{1}, \ldots, g_{p}$ be elements of $P$, the stabilizer of $X_{0}$ in $G$, such that $g_{i}\left(X_{1}\right)=Y_{i}$ for $i=1, \ldots, p$, and $\Delta_{i}=g_{i} \Delta g_{i}^{-1}, i=0, \ldots, p$. 
Then the group $\Gamma$ generated by $\Delta_{0}, \ldots, \Delta_{p}$ is discrete and is the free product of the "cusp subgroups" $\Delta_{i}$. Further, the fundamental domain of $\Gamma$ is $\bigcap_{i=0}^{p} g_{i}(F)=\bigcup_{i=0}^{p} g_{i}(L)$. Since $\operatorname{vol}(L)<\infty$ (see [S2, p. 89]) we deduce that $\Gamma$ is a lattice. Indeed, if $g_{i} \in S L_{2}(k), \Gamma$ is a congruence subgroup of $S L_{2}(k[t])$ (see [S2, Exercise 5, p. 88]) and, in particular, is arithmetic. But small deformations of the $g_{i}$ 's still satisfy the assumptions and will therefore give other lattices. "Most" of them are, of course, nonarithmetic.

It turns out that a combination of the two constructions above, give essentially all lattices.

THEOREM 2. Let $\Gamma$ be a lattice in $G$. Then $\Gamma$ has a finite index subgroup $\Gamma_{1}$ such that $\Gamma_{1}=*_{i=1}^{h} \Delta_{i} * F_{l}$, where $\Delta_{i}, i=1, \ldots, h$ are lattices in unipotent radicals $U_{i}$ of minimal parabolic subgroups $B_{i}$, and $F_{l}$ is a Schottcky free group on l generators, $l<\infty$. (If $h \geq 1$ then $F_{l}$ is of infinite covolume.)

The theorem extends Ihara's Theorem (cf. [I or S2, p. 82]). It is deduced, using Bass-Serre theory of groups acting on trees [S2], from the following theorem:

THEOREM 3. Let $\Gamma$ be a lattice in $G$. Then $X / \Gamma$ is a union of a finite graph with finitely many infinite lines.

This theorem is an analog of the description of the fundamental domain of lattices in rank one semisimple real Lie groups as given by Garland and Raghunathan in [GR]. In particular it shows the finiteness of the number of "cusps." Our method basically imitates that of [GR], but at various points their Lie algebra arguments (which are not that convenient to carry out in characteristic $p$ ) are replaced by more geometric combinatorial arguments similar to those of Eberlein [E].

Theorem 3 was proved by Serre in [S2, p. 106] for arithmetic lattices in $S L_{2}$ by completely different methods. The reader is still encouraged to look at the pictures there.

In [GR], the geometric description of the quotient manifold does not give much algebraic information on $\Gamma$. Here, due to Bass-Serre theory, it gives, as mentioned above, Theorem 2 from which we can deduce:

COROllary 4. If $\Gamma$ is a nonuniform lattice in $G$ (i.e. $h \geq 1$ ), then $\Gamma$ is not finitely generated.

This corollary was proved for arithmetic lattices in $S L_{2}$ by Serre [S2] and by $\mathrm{H}$. Behr and his students by a case-by-case study for arithmetic lattices in general $G$ (see [B] and the references therein).

Corollary 4 follows from Theorem 2 by observing that $\Delta=\Delta_{i}$ is a quotient of $\Gamma^{\prime}$ and it is not finitely generated. In fact $\Delta$ has an uncountable number of finite index subgroups while there are only countably many congruence subgroups. From this we deduced the following corollary which was conjectured by Serre [S1].

COROLlaRY 5. If $\Gamma$ is an arithmetic lattice in $G$, then $\Gamma$ does not have the congruence subgroup property.

For lattices in $S L_{2}$ it was proved by Serre in [S2]. 
Finally we mention that $G$ is embedded as a cocompact subgroup in $A=\operatorname{Aut}(X)$ so every lattice in $G$ is a lattice in $A$. But the general lattices in $A$ satisfy neither Theorem 2 nor Theorem 3. For more in this direction see [BL]. The key difference is the "local nilpotency" of $G$.

The author acknowledges useful suggestions and discussions with J. Bernstein, I. Piatetski-Shapiro, I. Rips and P. Sarnak.

\section{REFERENCES}

[B] H. Behr, Finite presentability of arithmetic groups over global function fields, Proc. Edinburgh Math. Soc. 30 (1987), 23-39.

[BL] H. Bass and A. Lubotzky (in preparation).

[BT] F. Bruhat and J. Tits, Groupes algébriques simples sur un corps locals, Proc. Conf. on Local Fields (T. A. Springer, ed.) (Driebergen) Springer-Verlag, New York, 1967, pp. 23-36.

[DM] P. Deligne and G. D. Mostow, Monodromy of hypergeometric functions and non lattice integral monodromy groups, Inst. Hautes Études Sci. Publ. Math. 63 (1986), 5-90.

[E] P. Eberlein, Lattices in spaces of non positive curvature, Ann. of Math. (2) 111 (1980), 435-476.

[GP] L. Gerritzen and M. van der Put, Schottky groups and Mumford curves, Lecture Notes in Math., vol. 817, Springer-Verlag, New York, 1980.

[GPS] M. Gromov and I. Piatetski-Shapiro, Non-arithmetic groups in Lobachevsky spaces, Inst. Hautes Études Sci. Publ. Math. 66 (1988), 93-103.

[GR] H. Garland and M. S. Raghunathan, Fundamental domains for lattices in (R)-rank 1 semisimple Lie groups, Ann. of Math. (2) 92 (1970), 279-326.

[I] Y. Ihara, On discrete subgroups of the two by two projective linear group over $\mathfrak{p}$-adic fields, J. Math. Soc. Japan 18 (1966), 219-235.

[Ka] D. A. Kazdhan, Some applications of the Weil representation, J. Analyse Math. 32 (1977), 235-248.

[L1] A. Lubotzky, Group presentation, p-adic analytic groups and lattices in $S L_{2}(C)$, Ann. of Math. (2) 118 (1983), 115-130.

[L2] L Lattices in rank one semisimple Lie groups over local fields (in preparation).

[Ma] G. A. Margulis, Arithmeticity of irreducible lattices in the semi-simple groups of rank greater than 1, Invent Math. 76 (1984), 93-120.

[Mi] J. J. Millson, On the first Betti number of constant negative curved manifold, Ann. of Math. (2) 104 (1976), 235-247.

[M1] G. D. Mostow, Strong rigidity of locally symmetric spaces, Ann. of Math. Stud., no. 78, Princeton Univ. Press, Princeton, N.J., 1973.

[M2] _ - On a remarkable class of polyhedra in complex hyperbolic space, Pacific J. Math. 86 (1980), 171-276.

[R1] M. S. Raghunathan, On the congruence subgroup problem, Inst. Hautes Études Sci. Publ. Math. 46 (1976), 107-161.

[R2] __, On the congruence subgroups problem. II, Invent. Math. 85 (1986), 73-117.

[S1] J.-P. Serre, Le problème des groupes de congruence pour $S L_{2}$, Ann. of Math. (2) 92 (1970), 489-527.

[S2] _ _ Trees, Springer-Verlag, New York, 1980.

[Z] R. Zimmer, Ergodic theory and semi-simple groups, Birkhäuser, Boston, 1984.

Institute OF Mathematics, Hebrew University, Jerusalem 91904, IsRael 